\title{
Evaluation of a College-Level Nutrition Course With a Teaching Kitchen Lab
}

\author{
Susana L. Matias, PhD; Jazmin Rodriguez-Jordan, PhD, RD; Mikelle McCoin, MPH, RD
}

\begin{abstract}
Objective: To evaluate the impact of a college nutrition course with a teaching kitchen lab on students' attitudes, self-efficacy, and behaviors about healthful eating and cooking.

Methods: Preintervention and postintervention design, and anonymous online survey of sociodemographic information and students' attitudes and self-efficacy about consuming fruits, vegetables, and whole grains and about cooking, self-reported intake, and cooking behaviors.

Results: Two-hundred and fourteen participants enrolled in the study during 5 semesters. Of these, $171(80 \%)$ had complete pretest and posttest data. Attitudes and self-efficacy scores about consuming fruits, vegetables, whole grains, and cooking were significantly higher in the posttest (vs pretest; all $P<0.0001$ ). Self-reported intake of fruits $(P<0.0001)$ and vegetables $(P=0.0006)$ also increased. Cooking frequency increased $(P<0.0001)$, skipping meals frequency decreased $(P<0.0001)$, whereas no significant changes were observed for eating out, take-out, or premade meals frequency.

Conclusions and Implications: A college nutrition course with a teaching kitchen lab could improve healthful eating and promote cooking in young adults.

Key Words: college students, teaching kitchen, nutrition course, cooking, diet (J Nutr Educ Behav. 2021;53:787-792.)
\end{abstract}

Accepted February 9, 2021. Published online March 18, 2021.

\section{INTRODUCTION}

Emerging adulthood, a period defined as 18-25 years of age, is characterized by important life transitions, such as going to college or moving away from home and becoming more independent in daily life decisions. However, many young adults may lack the skills to manage their time and maintain a healthy balance in the midst of their new independent life and academic or job responsibilities. Among the new tasks, young adults need to assume is the preparation or procurement of their own meals, which can be challenging for some. In a diverse sample of community college and university students in the Midwest, $37 \%$ to $46 \%$ reported experiencing time constraints affecting diet-related behaviors. ${ }^{1}$ Other factors limiting college students' ability to prepare and consume healthy meals include financial constraints, the widespread presence of unhealthy foods, lack of culinary knowledge and skill, and inadequate access to healthy food options. ${ }^{2,3}$ These barriers can lead to a consistent suboptimal diet and consequently weight gain, particularly at the beginning of college life. ${ }^{4}$ At the national level, only 5\% of college students reported eating 5 or more servings of fruits and vegetables per day, and $42 \%$ of them reported an unhealthy weight for their height (ie, body mass index outside of the $18.5-24.9 \mathrm{~kg} / \mathrm{m}^{2}$ range). ${ }^{5}$ In contrast, improved food knowledge and education, meal planning, and cooking more frequently

Department of Nutritional Sciences and Toxicology, University of California Berkeley, Berkeley, CA

Conflict of Interest Disclosure: The authors have not stated any conflicts of interest.

Address for correspondence: Susana L. Matias, PhD, Department of Nutritional Sciences and Toxicology, University of California Berkeley, 225 Morgan Hall, Berkeley, CA 94720; E-mail: slmatias@berkeley.edu

(C) 2021 The Authors. Published by Elsevier Inc. on behalf of Society for Nutrition Education and Behavior. This is an open access article under the CC BY-NC-ND license (http://creativecommons.org/licenses/by-nc-nd/4.0/)

https://doi.org/10.1016/j.jneb.2021.02.004 and with greater skills facilitated healthier eating and weight in this population. ${ }^{3,6}$

The period of emerging adulthood is also a time for self-identity development, $^{7}$ offering an opportunity for establishing long-term health behavior patterns. In a longitudinal study, participants who were engaged in food preparation as emerging adults were more likely to prepare meals including vegetables, have a higher intake of fruits and vegetables, and less sugar-sweetened beverage and fast-food consumption in their mid-to-late 20s. ${ }^{8}$ Developing adequate cooking skills in young adulthood has also been associated with more frequent meals with vegetables and less frequent fast-food consumption 10 years later. ${ }^{9}$ This long-term impact underlines the importance of designing programs to improve food behaviors and practices of young adults. The objective of this study was to assess changes in attitudes, self-efficacy, and behaviors related to healthy eating and cooking among college students who enrolled in a nutrition course with an integrated teaching kitchen lab in a large public university. 


\section{METHODS}

\section{Study Design}

This pre-post intervention study was conducted to evaluate changes in diet and meal preparation behaviors among college students after taking an undergraduate nutrition course with a teaching kitchen lab. The intervention consisted of a 14-week (semester-long) course that was developed in support of on-campus efforts to address food insecurity among students. The rationale for a nutrition and cooking course as a response to food insecurity relates to the less healthy diet, ${ }^{10-12}$ lower cooking self-efficacy ${ }^{13}$ and food agency, ${ }^{12}$ and less cooking or food preparation $^{12,13}$ reported by foodinsecure college students when compared with their food-secure counterparts.

\section{Intervention}

The 2-unit elective course relied on principles from the Social Cognitive Theory ${ }^{14}$ to address students' knowledge, attitudes, self-efficacy, skills, and behaviors regarding food procurement and preparation. Hands-on education provided in a teaching kitchen allowed students to learn through observation, ${ }^{15}$ address social norms, and engage in skill-building activities. Students attended a weekly 50 -minute lecture led by 1 of the investigators (J.R.J.), followed by a required 2-hour faculty and/or graduate student-led cooking lab in a 9-station built-in teaching kitchen on campus. The content of the lectures included basic nutrition and cooking concepts, meal planning, and food budgeting. Students learned about nutrients and their food sources, Dietary Guidelines for Americans, ${ }^{15}$ calculation of energy and nutrient needs, reading food labels, and mindful eating. An in-depth discussion of food insecurity among college students, with representation from various local resources (eg, CalFresh County Educator) was also covered. Cooking concepts reviewed included food storage and food safety, and various cooking methods using different kitchen equipment such as the stovetop, oven, and microwaves. Cost-saving techniques such as meal planning, budgeting, and purchasing foods in season or with coupons were also discussed. During the cooking lab sessions, students had access to basic kitchen equipment including blenders, food processors, cookware, bakeware, and cutlery. Students worked in pairs at each station to promote problem solving and minimize perceived barriers to cooking and trying new techniques. The recipes included affordable and nutritious ingredients and were easy to follow and quick to prepare to promote self-efficacy for cooking. They were selected to teach students how to cook with vegetables, grains, legumes, and animal-based proteins, although, plant-based meals were emphasized. In addition, students completed several small and 2 major projects: (1) a grocery shopping activity to understand the relationship between the nutritional quality of foods and cost, and (2) a 7-day meal plan with specific meal and food group criteria on a $\$ 50$ budget, and an analysis of its nutrients.

\section{Participants and Recruitment}

In 2017, the overall undergraduate population in this large public university was 30,574 , of which $52 \%$ were female and $21 \%$ of students had transferred from a community college. In addition, 39\% self-identified as Asian, 25\% as White, and 13\% were international students. The nutrition course was an elective class open for enrollment to undergraduate students of any academic standing (eg, freshman, senior). However, priority was given to students who were deemed potentially at risk of food insecurity, which was determined by the instructor on the basis of students' answers to a 4-question (eg, CalFresh-Supplemental Nutrition Assistance Program in California-eligibility) screener that was completed when students attempted to enroll in the class. In addition, the course was advertised at locations believed to be visited by high-risk students, such as the Center for Educational Equity and Excellence which serves many nontraditional students, firstgeneration students, students with dependents, or from underrepresented groups) and the campus food pantry. This study reflects data collected over 5 semesters (from Fall 2017 to Fall 2019). The first cooking lab session was dedicated to explaining the research activities, inviting students into the study, and reviewing the consent form. All study participants signed the consent form before providing any data. The University of California Berkeley Institutional Review Board approved the study protocol before its implementation.

\section{Instruments, Measures, and Procedures}

This study used a previously validated survey ${ }^{16}$ to measure attitudes and self-efficacy about the consumption of fruits and vegetables and selfreported usual fruit and vegetable intake and meal preparation behaviors. Previous content and convergent validation of Clifford et $\mathrm{al}^{16}$ survey was conducted by involving an expert panel of researchers, and by keeping in the final version only items that showed an interitem correlation $>0.70$, respectively. Reliability of that survey was determined by test-retest conducted 14 days apart with resulting correlations $>0.70 .^{16}$ Using Likert scales, the students indicated their agreement or disagreement with statements such as I feel that fruit is [vegetables are] not tasty, and Cooking is hard to assess attitudes, or I can find ways to eat fruit [vegetables] at every meal, and I can follow a recipe to assess self-efficacy. Similar scales using the same items used for fruits and vegetables but applying them to whole grains instead (eg, I can find ways to eat whole grains at every meal) were developed to assess attitudes and selfefficacy for increasing intake of whole grains. The attitudes and selfefficacy scales for each food group (eg, fruits) had 9 items each; the cooking attitudes scale had 6 items and the cooking self-efficacy scale had 4 items. Each item scored from 5 = Strongly agree (or Extremely confident) to $1=$ Strongly disagree (or Not at all confident); reverse coding was implemented for negative items before calculating total scores. Total scale range was $9-45$ points for attitudes and self-efficacy about food groups, 6-30 points for attitudes 
toward cooking, and 4-20 points for cooking self-efficacy. The reliability (internal consistency) of these scales in the study sample was deemed satisfactory ${ }^{17}$ (Cronbach alpha coefficients ranged from 0.70 to 0.94 ).

The behavior questions in the survey consisted of participants' selfreport of their usual daily intake of cups of fruits and vegetables, and ounces of whole grain. ${ }^{16}$ The number of cups of fruits and vegetables was measured in increments of 0.5 cups, from none $(0)$ to $>5$ cups (no one selected this highest option). Similarly, the number of ounces of whole grains was measured in $0.5 \mathrm{oz}$ increments, from none to $>5 \mathrm{oz}$. Written and visual examples of these measurement units were provided. Students also reported the number of times per week they cooked or prepared their own meals, ate premade meals, ate out or ate take-out, ate in a dining hall on campus, and skipped meals, for each of the 3 meals a day. Scoring for each of these meal-related behaviors could range from 0 to 21 times per week.

Participants completed these pretest and posttest assessments by responding to an anonymous online survey at the beginning and at the end of the semester. Students were provided a unique study identifier that they had to enter into the online survey as the identifier for linking the pretest and posttest assessments. Besides questions about the study outcomes, the online survey also asked about sociodemographic characteristics (eg, age, gender, race, or ethnicity). Students self-identified with ethnic/race groups from a list including White/Caucasian, African American/Black, Hispanic or Latino, Asian, Pacific Islander, Native American, other (specify), or they could opt not to answer.

\section{Data Analysis}

Descriptive statistics (ie, frequencies and percentages) were used to characterize the sample characteristics. Normality of continuous data was assessed using graphical methods (eg, histograms) and shape parameters (eg, skewness). For normally distributed continuous data (ie, attitudes and self-efficacy scores), means and standard deviations were calculated for each time point (ie, pre and post), and paired $t$ test $(\Delta=$ post - pre $)$ was used to determine statistical significance. In addition, the corresponding Cohen $d$ effect size ([Meanpost - Mean $\left.\left._{\text {pre }}\right] / \mathrm{SD}_{\text {pool }}\right)^{18}$ was calculated. For noncontinuous data, such as count data (ie, dietary intake data), or non-normally distributed continuous data (ie, meal-related behaviors), the median and interquartile range (IQR) were calculated for each time point, and the Wilcoxon signed-rank test ${ }^{19}$ was used to test if the difference ( $\Delta=$ post - pre) was statistically significant. All hypotheses testing were 2 -sided, and the significance level was set to $5 \%$; because of multiple testing this resulted in a cutoff of $P<0.0031$ for significance after the Bonferroni correction $(\alpha / k$, where $k=$ no. of hypothesis tests) was applied. All statistical analyses were conducted using SAS software (version 9.4, SAS Institute Inc, 2013).

\section{RESULTS}

Of 216 students enrolled in the course, 214 students enrolled in the study and completed 404 online pre- and posttest surveys. Of these surveys, 21 were excluded for not including a study ID, as that did not allow linkage of pretest and posttest assessments. Of the 383 surveys remaining, pretest and posttest surveys were linked for 171 participants; these participants ( $80 \%$ of those enrolled) comprised the analytic sample for this analysis. Participants included in this analysis were statistically similar to those who remained unpaired and were not included $(n=41)$ with regard to age $(P=0.74)$, gender $(P=0.25)$, race/ethnicity $(P=0.25)$, academic standing $(P=0.44)$, housing arrangement $(P=0.74)$, previous or current nutrition coursework $(P=0.24)$, baseline food security $(P=0.36)$ and intake of fruits $(P=0.77)$ and whole gains $(P=0.73)$; however, baseline intake of vegetables was lower among those included in the analysis (median [IQR], 1.0 [0.5-1.5]) compared with those not included (2.0 [1.0-2.5]; $P<$ 0.001 ). Table 1 shows sample characteristics on the basis of the baseline (pretest) survey data. Most participants were female students, self-identified as
Table 1. Sample Characteristics $(n=171)$

\begin{tabular}{lc} 
Characteristic & $\mathbf{n}(\%)$ \\
Age, y & \\
$18-20$ & $89(52.0)$ \\
$21-23$ & $74(43.3)$ \\
$\geq 24$ & $8(4.7)$ \\
Gender & \\
Female & $107(62.6)$ \\
Male & $62(36.3)$ \\
Other & $2(1.2)$ \\
Race/ethnicity & \\
Non-Hispanic White & $19(11.1)$ \\
Non-Hispanic Asian & $90(52.6)$ \\
Hispanic & $42(24.6)$ \\
Non-Hispanic Other & $20(11.7)$ \\
Academic standing & \\
Freshman & $15(8.8)$ \\
Sophomore & $29(17.0)$ \\
Junior & $49(28.7)$ \\
Senior & $74(43.3)$ \\
Other & $4(2.3)$ \\
Housing & \\
Off-campus & $138(80.7)$ \\
On-campus & $33(19.3)$ \\
\hline
\end{tabular}

Non-Hispanic Asians, and lived offcampus. In addition, 52\% reported food insecurity, and $51 \%$ had no previous nutrition coursework.

Table 2 shows the outcomes measured at each time point and the $P$ value for the hypothesis testing of the difference $(\Delta=$ post - pre). All attitudes and self-efficacy scores were significantly higher in the posttest compared with the pretest. The mean difference for attitudes toward vegetables was 4.2 (95\% confidence interval, 3.3-5.0), for attitudes toward fruits, it was 2.4 (1.7-3.2), for attitudes toward whole grains, it was 2.9 (2.1-3.7), and for attitudes toward cooking it was 4.0 (3.5-4.5). The mean difference for self-efficacy for increasing intake of vegetables was $7.2(6.0-8.4)$, for self-efficacy for eating more fruits, it was $5.4(4.3-6.5)$, for self-efficacy for increasing consumption of whole grains, it was 4.3 (3.1-5.4), and for self-efficacy for cooking it was 4.5 (4.0-4.9). With regard to dietary intake count data, the number of cups of vegetables and fruits consumed was significantly higher in the posttest compared with the pretest (median $\Delta=0.5$ [IQR, 0.0 -1.0] for vegetables; median $\Delta=0.0$ 
Table 2. Diet and Cooking Related Attitudes, Self-Efficacy, and Behaviors Among College Students in a Public University $(n=171)$

\section{Outcome \\ Vegetables attitudes score \\ Fruits attitudes score \\ Whole grains attitudes score \\ Cooking attitudes score ${ }^{d}$ \\ Vegetable self-efficacy score ${ }^{d}$ \\ Fruit self-efficacy score ${ }^{d}$ \\ Whole grain self-efficacy score ${ }^{d}$ \\ Cooking self-efficacy score ${ }^{d}$}

\begin{tabular}{c} 
Pretest \\
\hline Mean \pm SD \\
$3.7 \pm 4.9$ \\
$5.0 \pm 4.1$ \\
$4.1 \pm 4.3$ \\
$1.0 \pm 3.5$ \\
$31.3 \pm 8.1$ \\
$31.1 \pm 7.5$ \\
$35.0 \pm 8.0$ \\
$11.9 \pm 2.8$
\end{tabular}

Median (Interquartile Range)

$1.0(0.5-1.5)$
$1.0(0.5-2.0)$
$2.5(2.0-4.0)$
$7.0(3.0-11.0)$
$3.0(0.0-5.0)$
$4.0(2.0-7.0)$
$0.0(0.0-0.0)$
$4.0(2.0-7.0)$

Posttest

Mean \pm SD

$7.9 \pm 5.3$
$7.4 \pm 4.5$
$7.0 \pm 5.0$
$5.0 \pm 3.1$
$38.5 \pm 7.0$
$36.5 \pm 6.9$
$39.2 \pm 7.1$
$16.4 \pm 2.5$

Effect Size $^{a}$
0.83
0.57
0.62
1.21
0.96
0.76
0.58
1.66

$\begin{aligned} & \boldsymbol{P}^{\mathrm{b}} \\ &<0.0001^{\mathrm{c}}<0.0001^{\mathrm{c}} \\ &<00.0001^{\mathrm{c}} \\ &<0.0001^{\mathrm{c}} \\ &<0.0001^{\mathrm{c}} \\ &<0.0001^{\mathrm{c}} \\ &<0.0001^{\mathrm{c}} \\ &<0.0001^{\mathrm{c}}\end{aligned}$

\begin{abstract}
Cups of vegetables
Cups of fruit

Ounces of whole grain

Times/wk cooked

Times/wk ate premade meals

Times/wk ate out or take-out

Times/wk ate at dining hall

Times/wk skipped meals
\end{abstract}

\section{Median \\ (Interquartile Range)}

$$
\begin{gathered}
1.5(1.0-2.0) \\
1.0(0.5-2.0) \\
3.0(2.0-4.0) \\
10.0(6.0-14.0) \\
2.0(0.0-5.0) \\
4.0(2.0-6.0) \\
0.0(0.0-0.0) \\
3.0(0.0-5.0)
\end{gathered}
$$

\begin{tabular}{lr}
- & $<0.0001^{e}$ \\
- & $0.0006^{e}$ \\
- & $0.4793^{e}$ \\
- & $<0.0001^{e}$ \\
- & $0.3368^{e}$ \\
- & $0.0051^{e}$ \\
- & $0.8240^{e}$ \\
- & $<0.0001^{e}$ \\
\hline
\end{tabular}

${ }^{\mathrm{a}}$ Cohen $d=\left(\right.$ Mean $_{\text {post }}-$ Mean $\left._{\text {pre }}\right) / \mathrm{SD}_{\text {pool }}$. Calculated only for normally distributed outcome data; ${ }^{\mathrm{b}}$ Bonferroni adjusted $P$ value for significance: $0.05 / 16=0.0031 ;{ }^{c} t$ test for paired data $\left(\Delta=\right.$ post - pre); ${ }^{d} n=170$ because of missing value; ${ }^{e}$ Wilcoxon signedrank test for non-normally distributed paired data $(\Delta=$ post - pre).

[-0.5 to 1.0$]$ for fruits); however, no significant changes were observed for ounces of whole grains (median $\Delta=0.0 \quad[-1.0$ to 1.0$])$. Regarding meal-related behaviors, the number of times per week the participants ate meals they cooked increased in the posttest compared with the pretest (median $\Delta=2[-1$ to 5$]$ ), whereas the number of times they skipped meals decreased (median $\Delta=-1$ [ -4 to 0$]$ ). After applying the Bonferroni correction to the $P$ values, no significant changes were evident for the number of times participants ate premade meals (median $\Delta=0[-2$ to 1$]$ ), ate out or take-out (median $\Delta=0[-3$ to 1]) or ate in a dining hall on campus (median $\Delta=0[0,0]$ ).

\section{DISCUSSION}

Overall, this study found that an elective nutrition course with a teaching kitchen lab may improve dietary and cooking behaviors. Because behavioral change is the final goal of nutrition education it is important to identify potential elements of this undergraduate course that may have motivated behavioral change and could contribute to intervention effectiveness in future efforts involving young adults. First, the peer support and collaboration experienced by students when working in rotating pairs during the weekly cooking labs may have encouraged skill development. $^{20}$ Skill building and skill reinforcement, other key experiential drivers of behavior change, ${ }^{20}$ may have been more likely to occur in this semester-long intervention that offered enough practice opportunities for advancement of skills.

The study findings are consistent with those from a systematic review that highlighted the positive impact of cooking classes on diet and cooking confidence $^{21}$; however, most participants in the studies included in that review were middle-aged or older adults with a clinical condition. Nutrition and cooking interventions with college students (or young adults) are scarce, which limits the ability to compare this study's findings with previous relevant evidence, but also highlights the evidence gap in this age group. A similar intervention was conducted in a Brazilian university. ${ }^{22}$ Bernardo and collaborators ${ }^{22}$ used an experimental design to evaluate a nutrition and culinary kitchen program implemented over 6 weeks, 3 hours weekly, and based on 5 handson cooking classes and 1 food market visit. Their cooking-based curriculum had a positive impact on self-efficacy regarding cooking and produce consumption in the college students who participated, but did not affect cooking frequency. ${ }^{22}$ Another intervention in a South Dakota college consisted of a series of 3 cooking classes focused on simple, healthy recipes with inclusion of food safety education and nutrition instruction. ${ }^{23}$ That intervention resulted in increased nutrition knowledge, and cooking confidence and ability, but it did not change the frequency of food consumption or cooking among students. ${ }^{23}$ These findings seem to support the point made above that longer classes (exposure), like the 14-week nutrition and cooking course evaluated in this study, may promote not only skill building but also skill reinforcement, allowing translation from self-efficacy to actual behavior change.

Interpretation of the effect sizes observed in this study ${ }^{18}$ would 
suggest that the nutrition course could have a larger effect on attitudes and self-efficacy for vegetable consumption and cooking compared with those for fruit and whole-grain intake. This finding may relate to greater room for improvement, as seen in the pretest scores. The inclusion of vegetables in every recipe cooked at the teaching kitchen lab may have also played a role in these findings. More importantly, behavioral theory hypothesizes improvements in attitudes and self-efficacy facilitate behavioral changes, ${ }^{24,25}$ which may explain what was observed in these results.

Of interest, attitudes and self-efficacy for increased consumption of whole grains improved, yet self-reported intake of this food group did not change. It is important to note that while the scales about whole grains were developed on the basis of similar scales for fruits and vegetables, they did not follow the same validation procedures conducted by Clifford et $\mathrm{al}^{16}$; therefore, their validity is uncertain. Moreover, the nutrition course curriculum did not emphasize whole grains, to the same extent as vegetables, in the recipes cooked in the kitchen teaching lab sessions. Future programming could benefit from including more recipes using whole grains as ingredients.

Overall, study findings may indicate that using the format of a regular graded, elective undergraduate course that includes hands-on nutrition education and a teaching kitchen lab may influence attitudes, self-efficacy, and behaviors in this young adult population. Because cooking experiences during emerging adulthood can have long-lasting positive effects, ${ }^{8,9}$ development and evaluation of similar nutrition courses with an integrated teaching kitchen lab in undergraduate settings is needed.

Although the findings from this work are intriguing, this study has several limitations, with the main one being the lack of a control or comparison group. This limitation reduces the ability to attribute the observed changes to the nutrition course, as they could be due to other exposures besides the intervention. Other limitations of this study include the use of a convenience sample, which limits generalizability of study findings, and the use of selfreport to assess usual dietary behaviors, which could have introduced reporting bias. Future studies could include objective measures, such as noninvasive optical methodologies to measure skin carotenoid, a biomarker for fruit and vegetable intake. ${ }^{26}$ Another limitation was the loss of participants for whom the preand postsurvey linkage was not possible because of missing study ID $(n=41)$. Comparison between those included vs those not matched did not indicate significant differences in sociodemographic characteristics, but vegetable intake differed at baseline. In addition, the high participation rate $(99 \%$ among those who enrolled in the course) may relate to 1 of the investigators was the instructor for this class. However, study participation rate in a subsequent semester (not reported here), with a different noninvestigator instructor, was also high $(80 \%)$. Strengths of this study were the inclusion of a socioeconomically and racially diverse sample of college students (although not completely reflective of the university's student body), and the use of an anonymous online survey which likely reduced interviewer effects and social desirability bias. ${ }^{27}$

\section{IMPLICATIONS FOR RESEARCH AND PRACTICE}

Chance cannot be ruled out as a potential explanation for the positive results given the current study used a preexperimental design. Further evaluation of the impact of this, and future, nutrition and cooking course (s) will need to include a control group, which would allow examination of a causal-effect relationship. ${ }^{28}$ Furthermore, future studies would benefit from including in their design follow-up assessments that could also provide evidence about the sustainability of any end line effects. Although costly and logistically more complicated, longitudinal evaluation studies that follow students beyond their college years and include clinical outcomes (eg, obesity) are also needed. These studies could provide evidence of sustained and potential long-term effects of nutrition and cooking interventions in college settings. Exposing young adults to food preparation activities has been shown to have lasting positive effects in establishing healthful behaviors during this critical transitional period in life. ${ }^{8,9}$ Thus, building strong evidence about the impact of a hands-on nutrition and teaching kitchen course in an optimal setting, such as higher education institutions, is warranted.

\section{ACKNOWLEDGMENTS}

The authors would like to thank the University of California Berkeley, Basic Needs Committee for institutional and financial support; Ruth Schrager, MPH, RD; and Aly Thompson, MPH, RD for their early involvement with the course and curriculum; Ryan Farquhar for leading some of the cooking lab sessions; and Joseph Napoli, PhD for initial support with ethical approvals.

\section{REFERENCES}

1. Pelletier JE, Laska MN. Balancing healthy meals and busy lives: associations between work, school, and family responsibilities and perceived time constraints among young adults. J Nutr Educ Behav. 2012;44:481-489.

2. Murray DW, Mahadevan M, Gatto K, et al. Culinary efficacy: an exploratory study of skills, confidence, and healthy cooking competencies among university students. Perspect Public Health. 2016;136:143-151.

3. Sogari G, Velez-Argumedo C, Gómez MI, Mora C. College students and eating habits: a study using an ecological model for healthy behavior. Nutrients. 2018;10:1823

4. Crombie AP, Ilich JZ, Dutton GR, Panton LB, Abood DA. The freshman weight gain phenomenon revisited. Nutr Rev. 2009;67:83-94.

5. American College Health Association. National College Health Assessment II: Reference Group Executive Summary Fall 2017. American College Health Association; 2018.

6. Hanson AJ, Kattelmann KK, McCormack LA, et al. Cooking and meal planning as predictors of fruit and vegetable intake and BMI in first-year 
college students. Int J Environ Res Public Health. 2019;16:2462.

7. Schwartz SJ, Zamboanga BL, Luyckx K, Meca A, Ritchie RA. Identity in emerging adulthood: reviewing the field and looking forward. Emerg Adulthood. 2013;1:96-113.

8. Laska MN, Larson NI, NeumarkSztainer D, Story M. Does involvement in food preparation track from adolescence to young adulthood and is it associated with better dietary quality? Findings from a 10-year longitudinal study. Public Health Nutr. 2012;15: 1150-1158.

9. Utter J, Larson N, Laska MN, Winkler M, Neumark-Sztainer D. Self-perceived cooking skills in emerging adulthood predict better dietary behaviors and intake 10 years later: a longitudinal study. J Nutr Educ Behav. 2018;50:494500 .

10. Martinez SM, Webb K, Frongillo EA, Ritchie LD. Food insecurity in California's public university system: what are the risk factors? J Hunger Environ Nutr. 2018;13:1-18.

11. Mirabitur E, Peterson KE, Rathz C, Matlen S, Kasper N. Predictors of college-student food security and fruit and vegetable intake differ by housing type. J Am Coll Health. 2016;64: 555-564.

12. Leung CW, Wolfson JA, Lahne J, Barry MR, Kasper N, Cohen AJ. Associations between food security status and dietrelated outcomes among students at a large, public midwestern university. $J$ Acad Nutr Diet. 2019;119:1623-1631.

13. Knol LL, Robb CA, McKinley EM, Wood M. Very low food security status is related to lower cooking self-efficacy and less frequent food preparation behaviors among college students. J Nutr Educ Behav. 2019;51:357-363.

14. Bandura A. Social cognitive theory: an agentic perspective. Annu Rev Psychol. 2001;52:1-26.

15. US Department of Health and Human Services, US Department of Agriculture. 2015-2020 Dietary Guidelines for Americans. 8th ed. US Department of Agriculture; 2015. https://health.gov/ our-work/food-nutrition/previousdietary-guidelines/2015. Accessed October 12, 2020.

16. Clifford D, Anderson J, Auld G, Champ J. Good Grubbin': impact of a TV cooking show for college students living off campus. J Nutr Educ Behav. 2009;41:194-200.

17. Bland JM, Altman DG. Statistics notes: Cronbach's alpha. BMJ. 1997;314:572.

18. Cohen J. A power primer. Psychol Bull. 1992;112:155-159.

19. Proudfoot JA, Lin T, Wang B, Tu XM. Tests for paired count outcomes. Gen Psychiatr. 2018;31:e100004.

20. Fredericks L, Koch PA, Liu A, Galitzdorfer L, Costa A, Utter J. Experiential features of culinary nutrition education that drive behavior change: frameworks for research and practice. Health Promot Pract. 2020;21:331-335.
21. Reicks M, Kocher M, Reeder J. Impact of cooking and home food preparation interventions among adults: a systematic review (2011-2016). J Nutr Educ Behav. 2018;50:148-172. e1.

22. Bernardo GL, Jomori MM, Fernandes AC, Colussi CF, Condrasky MD, Proença R. Positive impact of a cooking skills intervention among Brazilian university students: six months follow-up of a randomized controlled trial. Appetite. 2018;130:247-255.

23. Barr A, Hanson A, Kattelmann K. Effect of cooking classes on healthy eating behavior among college students. Top Clin Nutr. 2020;35:62-70.

24. Ajzen I. The theory of planned behavior. Organ Behav Hum Decis Processes. 1991;50:179-211.

25. Bandura A. Self-efficacy: toward a unifying theory of behavioral change. Psychol Rev. 1977;84:191-215.

26. Ermakov IV, Ermakova M, Sharifzadeh $M$, et al. Optical assessment of skin carotenoid status as a biomarker of vegetable and fruit intake. Arch Biochem Biophys. 2018;646:46-54.

27. Krumpal I. Determinants of social desirability bias in sensitive surveys: a literature review. Qual Quant. 2013;47: 2025-2047.

28. Hariton E, Locascio JJ. Randomised controlled trials - the gold standard for effectiveness research: study design: randomised controlled trials. BJOG. 2018;125:1716. 